\title{
USE OF LINEAR PROGRAMMING MODELS IN EXPERIMENTATION WITH PLANT NUTRIENTS
}

\author{
Mauro Brino Garcia ${ }^{1}$, Lucas Rezende Gomide ${ }^{2}$
}

(received: February 9, 2011; accepted: December 21, 2012)

\begin{abstract}
Nutrition is an important issue of plant cultivation and experimentation with plant nutrients is a supporting tool for agriculture. However, use of high purity grade reagents as nutrient sources can be expensive and increases the cost of an experiment. The objective of this study was to minimize the acquisition cost of high purity grade reagents in experiments on plant nutrient deficiency by using the missing element technique through linear programming models, and to generate recommendation tables for preparation of culture solutions, as well as to quantify gains through a simulated experiment. Two linear programming models were formulated containing concentration constraints for each nutrient in the culture solution. Model A was based on 16 reagents for preparation of the culture solution, while model B was based on 27 reagents, looking to increase choice options. Results showed that both models minimized the acquisition cost of reagents, allowing a 9.03\% reduction in model A and a $25.98 \%$ reduction in model B. The missing sulfur treatment proved the most costly for reagent acquisition while the missing nitrogen treatment proved the least costly. It was concluded that the formulated models were capable of reducing acquisition costs of reagents, yet the recommendations generated by them should be tested and checked for practical viability.
\end{abstract}

Key words: Operating research, optimization, missing element.

\section{USO DE MODELOS DE PROGRAMAÇÃO LINEAR APLICADO À EXPERIMENTAÇÃO NUTRICIONAL DE PLANTAS}

RESUMO: A nutrição vegetal é um importante aspecto no cultivo de espécies, sendo a experimentação nutricional uma ferramenta de suporte à agricultura. Entretanto, o uso de reagentes p.a. como fontes de nutrientes é caro e aumenta os custos do experimento. Assim, objetivou-se, com este trabalho, minimizar o custo de aquisição de reagentes p.a. em experimentos de deficiência nutricional de plantas, considerando a técnica do elemento faltante, por meio de modelos de programação linear, gerar tabelas de recomendação para a elaboração de soluções de cultivo e quantificar os ganhos por um experimento simulado. Dois modelos de programação linear foram formulados, contendo restrições de concentração para cada nutriente na solução de cultivo. O modelo A baseou-se na utilização de 16 reagentes na elaboração da solução de cultivo, já, o modelo B empregou 27, com o intuito de aumentar as opções de escolha. Os resultados mostraram que os dois modelos minimizaram o custo na aquisição de reagentes, com redução de $9,03 \%$ (modelo A) e 25,98\% (modelo B). O tratamento de omissão de Enxofre foi o mais oneroso para a aquisição de reagentes e o de omissão de Nitrogênio o menos oneroso. Conclui-se que os modelos formulados foram capazes de reduzir os custos na aquisição dos reagentes; porém deve-se testar as recomendações geradas pelos mesmos e verificar sua viabilidade prática.

Palavras-chave: Pesquisa operacional, otimização, elemento faltante.

\section{INTRODUCTION}

Soil is a biologically active layer of material resulting from complex transformations which in turn involve rock and mineral weathering, nutrient cycling, as well as biomass production and decomposition. It is thus the primary medium for plant growth (NOVAIS et al., 2007). A way of maintaining nutrient levels and soil stability is to use fertilization. However, suitable management of fertilization involves defining not only the correct dosages and sources of nutrients but also the best seasons and methods to apply liming and fertilizers to the soil (OLIVEIRA et al., 1991).
The learning process of soil-plant relationships calls for experiments with which to identify nutrient deficiencies in the target species (OLIVEIRA et al., 1991). The most widely used method in experiments on nutrient deficiency is the missing element technique, based on Liebig's law of the minimum (1840). For this experiment, high purity grade reagents (pure for analysis) were used as nutrient sources, that way ensuring maximum purity and precision although their prohibitive cost obstructs research.

Studies on mineral nutrition of plants are widely explored in literature (MAFFEIS et al., 2000; MARQUES

\footnotetext{
${ }^{1}$ Forest Engineer, MSc candidate in Soil Fertility and Plant Nutrition - Universidade Federal de Lavras/UFLA - Departamento de Ciência do Solo Cx.P. 3037 - 37200-00 - Lavras, MG, Brasil - garcia.floresta@gmail.com

${ }^{2}$ Forest Engineer, Professor PhD in Forest Engineering - Universidade Federal de Lavras/UFLA - Departamento de Ciências Florestais - Cx.P. 3037 -37200-00 - Lavras, MG, Brasil-lucasgomide@dcf.ufla.br
}

Cerne, Lavras, v. 19, n. 2, p. 255-261, abr./jun. 2013 
et al., 2004; SILVA et al., 2005; WALLAU et al., 2008). However, defining optimal nutrient formulations in connection with the acquisition cost of reagents is a subject not sufficiently explored by researchers, despite being critical to the reduction of such costs without affecting the development and conduction of an experiment.

Use of tools such as linear programming (LP) to assist the decision-making can contribute toward finding optimal responses (BERGER et al., 2003; RODRIGUEZ et al., 1985; SILVA et al., 2003), whenever possible, helping researchers solve complex problems. A line of research in the field of mathematical programming, LP is intended to maximize or minimize a function subject to linear constraints that delimit a feasible region (GOLDBARG; LUNA, 2005).

The objective of this study was to apply LP models to experimentation with plant nutrients in an attempt to minimize the acquisition cost of high purity grade reagents in experiments involving nutrient deficiency by using the missing element technique, and also to generate recommendation tables for preparation of culture solutions as well as to quantify gains through a simulated experiment.

\section{MATERIAL AND METHODS}

The base nutrient formulation to be optimized in this work was directed at experiments on nutrient deficiency (missing element technique), in which one nutrient is eliminated per treatment. The method generates a large number of combinations between reagents until the desired concentrations are attained. The LP mathematical model was thus based on the classic mixture problem (GOLDBARG; LUNA, 2005). The model developed for the relevant problem is provided as follows, where (1) is objective function, (2) are constraints on nutrient concentration and (3) is nonnegativity condition:

$$
\mathrm{FOmin}(\mathrm{R} \$) \sum_{i=1}^{n} P_{i} x_{i}
$$

subject to constraints

$$
\begin{aligned}
& \sum_{i=1}^{n k} R_{k i}=C_{k}, \forall_{k}\{1,2,3, \ldots, 13\} \\
& x_{i} \geq 0
\end{aligned}
$$

where,

$n$ - number of reagents used for providing nutrients tested in the experiment with the missing element technique.

$K$ - nutrients tested in the experiment with the missing element technique, including $\mathrm{N}, \mathrm{P}, \mathrm{K}, \mathrm{Ca}, \mathrm{Mg}, \mathrm{S}, \mathrm{Fe}, \mathrm{B}$, $\mathrm{Mn}, \mathrm{Zn}, \mathrm{Cu}$ and $\mathrm{Mo}$;

$P_{i}$ - price of each reagent, expressed as Reais per gram $\left(\mathrm{R} \$ . \mathrm{g}^{-1}\right)$;

$x_{i}$ - decision variable representing the quantity of reagent $i$ in gram (g) to be used in formulating one liter of culture solution;

$R_{k i}$ - quantity of nutrient $k$ available and present in reagent $i$, expressed as mass ratio of atomic weight of atoms of the nutrient present in the reagent to molecular mass of reagent;

$C_{k}$ - desired concentration of nutrient $k$ expressed as grams per liter of culture solution (g. $\left.{ }^{-1}\right)$ (PRADO, 2010).

Table 1 provides the price $\left(\mathrm{R} \$ . \mathrm{g}^{-1}\right)$ of every reagent being analyzed, relevant molecular formulations and which reagents are being tested in models A and B. Each reagent was correlated to a decision variable $\left(x_{i}\right)$ representing the quantity of reagent $k$ to be used in formulating one liter of culture solution. Reagent prices came from a quote obtained from a company selling laboratory products, on November 18, 2010.

The adopted reference table was obtained from Prado (2010), which provides $\mathrm{mg} . \mathrm{l}^{-1}$ concentrations of each nutrient $\left(C_{k}\right)$ in the culture solution, where: $\mathrm{N}$ (210.1011), P (30.9737), K (234.5898), Ca (200.3900), $\mathrm{Mg}$ (48.6100), S (64.1320), Fe (5.0103), B (0.5000), Mn (0.5025), Zn (0.0480), Cu (0.0189) and Mo (0.0107). With this information at hand, two LP models were developed, the first being termed model A and comprising 16 reagents, the second being termed model $\mathrm{B}$ and comprising 27 reagents in an attempt to increase model choice options.

Model constraints were formulated to define the required concentration of each nutrient in the culture solution, following the order: $\mathrm{N} ; \mathrm{P} ; \mathrm{K} ; \mathrm{Ca} ; \mathrm{Mg} ; \mathrm{S} ; \mathrm{Fe} ; \mathrm{B}$; $\mathrm{Mn} ; \mathrm{Zn} ; \mathrm{Cu}$ and Mo. To eliminate one nutrient and form a new nutrient-omitting treatment (missing element), it was necessary to reset parameter $C_{k}$ and thus form a new treatment scenario. The scenarios created in this study were analogous with experimentation treatments on plant nutrients. For solving the linear programming models, the commercial solver ILOG CPLEX 11 was used.

Cerne, Lavras, v. 19, n. 2, p. 255-261, abr./jun. 2013 
Table 1 - Number of reagents tested in Models A ( $\square)$ and B ( $\square$ ) with respective prices, formulas and decision variable.

Tabela 1 - Número de reagentes testados no Modelo $A(\square)$ e B ( $\square$ ) e seus respectivos preços, fórmulas e variável de decisão.

\begin{tabular}{|c|c|c|c|c|c|}
\hline \multicolumn{2}{|c|}{ Model } & \multirow{2}{*}{ Reagent } & \multirow{2}{*}{ Formula } & \multirow{2}{*}{ Decision variable } & \multirow{2}{*}{ Price $\left(\mathrm{R} \$ . \mathrm{g}^{-1}\right)$} \\
\hline $\mathrm{A}$ & $\mathrm{B}$ & & & & \\
\hline - & $\square$ & Potassium phosphate & $\mathrm{KH}_{2} \mathrm{PO}_{4}$ & $x_{1}$ & 0.01946 \\
\hline - & $\square$ & Potassium nitrate & $\mathrm{KNO}_{3}$ & $x_{2}$ & 0.07540 \\
\hline - & $\square$ & Calcium nitrate & $\mathrm{Ca}\left(\mathrm{NO}_{3}\right)_{2} \cdot 4 \mathrm{H}_{2} \mathrm{O}$ & $x_{3}$ & 0.04510 \\
\hline - & $\square$ & Magnesium sulfate & $\mathrm{MgSO}_{4} \cdot 7 \mathrm{H}_{2} \mathrm{O}$ & $x_{4}$ & 0.01720 \\
\hline - & $\square$ & Potassium chloride & $\mathrm{KCl}$ & $x_{5}$ & 0.05852 \\
\hline - & $\square$ & Calcium chloride & $\mathrm{CaCl}_{2} \cdot 2 \mathrm{H}_{2} \mathrm{O}$ & $x_{6}$ & 0.01550 \\
\hline - & $\square$ & Monoammonium phosphate & $\mathrm{NH}_{4} \mathrm{H}_{2} \mathrm{PO}_{4}$ & $x_{7}$ & 0.04810 \\
\hline - & $\square$ & Ammonium nitrate & $\mathrm{NH}_{4} \mathrm{NO}_{3}$ & $x_{8}$ & 0.07870 \\
\hline - & $\square$ & Ammonium sulfate & $\left(\mathrm{NH}_{4}\right)_{2} \mathrm{SO}_{4}$ & $x_{9}$ & 0.01036 \\
\hline - & $\square$ & Magnesium nitrate & $\mathrm{Mg}\left(\mathrm{NO}_{3}\right)_{2} \cdot 6 \mathrm{H}_{2} \mathrm{O}$ & $x_{10}$ & 0.11350 \\
\hline - & $\square$ & Boric acid & $\mathrm{H}_{3} \mathrm{BO}_{3}$ & $x_{11}$ & 0.02460 \\
\hline - & $\square$ & Manganese chloride & $\mathrm{MnCl}_{2} \cdot 4 \mathrm{H}_{2} \mathrm{O}$ & $x_{12}$ & 0.34250 \\
\hline - & $\square$ & Zinc chloride & $\mathrm{ZnCl}_{2}$ & $x_{13}$ & 0.10795 \\
\hline - & $\square$ & Copper chloride & $\mathrm{CuCl}_{2}$ & $x_{14}$ & 0.39092 \\
\hline - & $\square$ & Molybdic acid & $\mathrm{H}_{2} \mathrm{MoO}_{4} \cdot \mathrm{H}_{2} \mathrm{O}$ & $x_{15}$ & 0.72640 \\
\hline \multirow[t]{12}{*}{ - } & $\square$ & Iron chloride & $\mathrm{FeCl}_{3} \cdot 6 \mathrm{H}_{2} \mathrm{O}$ & $x_{16}$ & 0.08172 \\
\hline & $\square$ & Calcium sulfate & $\mathrm{CaSO}_{4} \cdot 2 \mathrm{H}_{2} \mathrm{O}$ & $x_{17}$ & 0.03090 \\
\hline & $\square$ & Sodium nitrate & $\mathrm{NaNO}_{3}$ & $x_{18}$ & 0,03300 \\
\hline & $\square$ & Potassium sulfate & $\mathrm{K}_{2} \mathrm{SO}_{4}$ & $x_{19}$ & 0.03800 \\
\hline & $\square$ & Sodium sulfate & $\mathrm{Na}_{2} \mathrm{SO}_{4}$ & $x_{20}$ & 0.02038 \\
\hline & $\square$ & Magnesium chloride & $\mathrm{MgCl}_{2} \cdot 6 \mathrm{H}_{2} \mathrm{O}$ & $x_{21}$ & 0.01800 \\
\hline & $\square$ & Monocalcium phosphate & $\mathrm{Ca}\left(\mathrm{H}_{2} \mathrm{PO}_{4}\right) \cdot 2 \mathrm{H}_{2} \mathrm{O}$ & $x_{22}$ & 0.05582 \\
\hline & $\square$ & Copper sulfate & $\mathrm{CuSO}_{4} \cdot 5 \mathrm{H}_{2} \mathrm{O}$ & $x_{23}$ & 0.03840 \\
\hline & $\square$ & Zinc sulfate & $\mathrm{ZnSO}_{4} \cdot 7 \mathrm{H}_{2} \mathrm{O}$ & $x_{24}$ & 0.03902 \\
\hline & $\square$ & Manganese sulfate & $\mathrm{MnSO}_{4} \cdot \mathrm{H}_{2} \mathrm{O}$ & $x_{25}$ & 0.07702 \\
\hline & $\square$ & Iron sulfate & $\mathrm{FeSO}_{4} \cdot 7 \mathrm{H}_{2} \mathrm{O}$ & $x_{26}$ & 0.02490 \\
\hline & $\square$ & Sodium phosphate & $\mathrm{NaH}_{2} \mathrm{PO}_{4}$ & $x_{27}$ & 0.03128 \\
\hline
\end{tabular}

An economic analysis of acquisition cost of reagents was carried out, comparing the reagent quantity recommended by Prado (2010) with the quantity found by models A and B in this work. For that, an experiment was simulated which lasted four months and whose experimental design consisted of 13 treatments (as used in the experiment on nutrient deficiency with the missing element technique), with 8 replicates and 4 vases per replicate. Each vase had a capacity of 3 liters and the culture solution was replaced every 7 days, according to variations in concentration due to plant absorption (TAIZ; ZEIGER, 2004). The strategy was to progress with the experiment, computing its cost with the culture solution.

\section{RESULTS AND DISCUSSION}

Model A selected virtually every reagent suggested by Prado (2010), except potassium nitrate $\left(x_{2}\right)$ and calcium nitrate $\left(x_{3}\right)$, using larger quantities of calcium chloride $\left(x_{6}\right)$, ammonium nitrate $\left(x_{8}\right)$ and magnesium sulfate $\left(x_{4}\right)$. It was noted that potassium nitrate $\left(x_{2}\right)$ and calcium nitrate $\left(x_{3}\right)$

Cerne, Lavras, v. 19, n. 2, p. 255-261, abr./jun. 2013 
were replaced with ammonium nitrate $\left(x_{8}\right)$ which, despite its higher unit cost, has a greater mass ratio of nitrogen and thus meets the nitrogen requirement at a lower cost. The quantities used of micronutrient-supplying reagents remained constant, as there are no alternative sources of these for replacement.

With a larger number of reagent options, model B selected the variables potassium phosphate $\left(x_{1}\right)$, potassium chloride $\left(x_{5}\right)$, calcium chloride $\left(x_{6}\right)$, monoammonium phosphate $\left(x_{7}\right)$, ammonium sulfate $\left(x_{9}\right)$, boric acid $\left(x_{11}\right)$, manganese chloride $\left(x_{12}\right)$, zinc chloride $\left(x_{13}\right)$, copper chloride $\left(x_{14}\right)$, molybdic acid $\left(x_{15}\right)$ iron chloride $\left(x_{16}\right)$, sodium nitrate $\left(x_{18}\right)$, potassium sulfate $\left(x_{19}\right)$, sodium sulfate $\left(x_{20}\right)$, magnesium chloride $\left(x_{21}\right)$, copper sulfate $\left(x_{23}\right)$, zinc sulfate $\left(x_{24}\right)$, manganese sulfate $\left(x_{25}\right)$, iron sulfate $\left(x_{26}\right)$, in which the most generally used were sodium nitrate $\left(x_{18}\right)$, calcium chloride $\left(x_{6}\right)$ and magnesium chloride $\left(x_{21}\right)$. Following results, the potassium nitrate $\left(x_{2}\right)$, calcium nitrate $\left(x_{3}\right)$ and ammonium nitrate $\left(x_{8}\right)$ used by Prado (2010) and in model A were replaced here with sodium nitrate $\left(x_{18}\right)$ due to low cost.

In both models $\mathrm{A}$ and $\mathrm{B}$, the main source of $\mathrm{Ca}$ was calcium chloride $\left(x_{6}\right)$. In model $\mathrm{B}$, magnesium chloride $\left(x_{21}\right)$ fully replaced magnesium sulfate $\left(x_{4}\right)$ and magnesium nitrate $\left(x_{10}\right)$ due to having only magnesium as nutrient and due to low cost.

Tables 2 and 3 provide recommendations for treatment composition (missing element), and values of stock solutions for each reagent selected in models A and $\mathrm{B}$ respectively.

Based on scaled quantities of each reagent, required costs per treatment were then generated for preparing one liter of culture solution, following the experiment with the missing element technique, as illustrated in Table 4.

By using the LP model clearly helped reduce the acquisition cost of reagents, with model A allowing savings of $9.03 \%$, using the same range of reagents suggested by Prado (2010), only with varying quantities of each reagent. In the treatment where $\mathrm{N}$ was omitted, no reduction was noted due to the poor choice of reagents without $\mathrm{N}$ in their composition. The treatment where $\mathrm{K}$ was omitted had a $12.55 \%$ cost reduction, mainly due to replacement of the Ca source, in which calcium chloride $\left(x_{6}\right)$ replaced calcium nitrate $\left(x_{3}\right)$. In addition, nine treatments attained a near to $10.50 \%$ reduction.

Table 2 - Composition of nutrient solutions ( $\mathrm{ml}$ of stock solution per liter of culture solution) according to model A.

Tabela 2 - Composição das soluções nutritivas ( $\mathrm{ml}$ da solução estoque por litro da solução de cultivo) de acordo com o modelo A.

\begin{tabular}{|c|c|c|c|c|c|c|c|c|c|c|c|c|c|}
\hline \multirow{2}{*}{ Reagent } & \multicolumn{13}{|c|}{ Treatment } \\
\hline & Cp. & $-\mathrm{N}$ & $-\mathrm{P}$ & $-\mathrm{K}$ & $-\mathrm{Ca}$ & $-\mathrm{Mg}$ & $-\mathrm{S}$ & $-\mathrm{Fe}$ & $-B$ & $-\mathrm{Mn}$ & $-\mathrm{Zn}$ & $-\mathrm{Cu}$ & -Mo \\
\hline & & & --- & 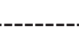 & --- & ----- & 11/1--- & --- & 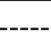 & --- & 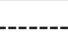 & & \\
\hline${ }^{1} \mathrm{KH}_{2} \mathrm{PO}_{4}$ & 1 & 1 & $\bullet$ & - & 1 & 1 & 1 & 1 & 1 & 1 & 1 & 1 & 1 \\
\hline${ }^{2} \mathrm{MgSO}_{4} \cdot 7 \mathrm{H}_{2} \mathrm{O}$ & 1 & 1 & 1 & 1 & 1 & • & - & 1 & 1 & 1 & 1 & 1 & 1 \\
\hline${ }^{3} \mathrm{KCl}$ & 2 & 2 & 2 & • & 2 & 2 & 2 & 2 & 2 & 2 & 2 & 2 & 2 \\
\hline${ }^{4} \mathrm{CaCl}_{2} \cdot 2 \mathrm{H}_{2} \mathrm{O}$ & 1 & 1 & 1 & 1 & - & 1 & 1 & 1 & 1 & 1 & 1 & 1 & 1 \\
\hline${ }^{5} \mathrm{NH}_{4} \mathrm{H}_{2} \mathrm{PO}_{4}$ & $\bullet$ & • & & 1 & - & $\bullet$ & $\bullet$ & $\bullet$ & $\bullet$ & - & - & - & • \\
\hline${ }^{6} \mathrm{NH}_{4} \mathrm{NO}_{3}$ & 2 & $\bullet$ & 2 & 1.87 & 2 & 1.47 & 1.47 & 2 & 2 & 2 & 2 & 2 & 2 \\
\hline${ }^{7}\left(\mathrm{NH}_{4}\right)_{2} \mathrm{SO}_{4}$ & $\bullet$ & - & - & - & $\bullet$ & 1 & - & $\bullet$ & - & - & - & $\bullet$ & $\bullet$ \\
\hline${ }^{8} \mathrm{Mg}\left(\mathrm{NO}_{3}\right)_{2} \cdot 6 \mathrm{H}_{2} \mathrm{O}$ & - & - & • & - & - & • & 1 & $\bullet$ & - & $\bullet$ & - & - & - \\
\hline${ }^{9} \mathrm{FeCl}_{3} \cdot 6 \mathrm{H}_{2} \mathrm{O}$ & 1 & 1 & 1 & 1 & 1 & 1 & 1 & • & 1 & 1 & 1 & 1 & 1 \\
\hline${ }^{10} \mathrm{H}_{3} \mathrm{BO}_{3}$ & 1 & 1 & 1 & 1 & 1 & 1 & 1 & 1 & $\bullet$ & 1 & 1 & 1 & 1 \\
\hline${ }^{11} \mathrm{MnCl}_{2} \cdot 4 \mathrm{H}_{2} \mathrm{O}$ & 1 & 1 & 1 & 1 & 1 & 1 & 1 & 1 & 1 & • & 1 & 1 & 1 \\
\hline${ }^{12} \mathrm{ZnCl}_{2}$ & 1 & 1 & 1 & 1 & 1 & 1 & 1 & 1 & 1 & 1 & $\bullet$ & 1 & 1 \\
\hline${ }^{13} \mathrm{CuCl}_{2}$ & 1 & 1 & 1 & 1 & 1 & 1 & 1 & 1 & 1 & 1 & 1 & • & 1 \\
\hline${ }^{14} \mathrm{H}_{2} \mathrm{MoO}_{4} \cdot \mathrm{H}_{2} \mathrm{O}$ & 1 & 1 & 1 & 1 & 1 & 1 & 1 & 1 & 1 & 1 & 1 & 1 & $\bullet$ \\
\hline
\end{tabular}

Stock solutions (g.l $\left.{ }^{-1}\right):{ }^{1} 136.0826 ;{ }^{2} 492.9439 ;{ }^{3} 186.396 ;{ }^{4} 735.0794 ;{ }^{5} 115.0244 ;{ }^{6} 300.1603 ;{ }^{7} 264.2766 ;{ }^{8} 512.7637 ;{ }^{9} 24.2499 ;{ }^{10} 2.86 ;{ }^{11} 1.81 ;{ }^{12} 0.099992$; ${ }^{13} 0.04001 ;{ }^{14} 0.019997$. Note: (Cp.) complete treatment. (-) Treatment without the relevant nutrient. (•) reagent not used in the relevant treatment.

Cerne, Lavras, v. 19, n. 2, p. 255-261, abr./jun. 2013 
Table 3 - Composition of nutrient solutions ( $\mathrm{ml}$ of stock solution per liter of culture solution) according to model B.

Tabela 3 - Composição das soluções nutritivas ( $m l$ da solução estoque por litro da solução de cultivo) de acordo com o modelo B.

\begin{tabular}{|c|c|c|c|c|c|c|c|c|c|c|c|c|c|}
\hline \multirow{2}{*}{ Reagent } & \multicolumn{13}{|c|}{ Treatment } \\
\hline & $\mathrm{Cp}$. & $-\mathrm{N}$ & $-\mathrm{P}$ & $-K$ & $-\mathrm{Ca}$ & $-\mathrm{Mg}$ & $-S$ & $-\mathrm{Fe}$ & $-\mathrm{B}$ & $-\mathrm{Mn}$ & $-\mathrm{Zn}$ & $-\mathrm{Cu}$ & $-\mathrm{Mo}$ \\
\hline & & & & & & $---\mathrm{ml}$ & ----- & 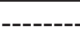 & & - & & & \\
\hline${ }^{1} \mathrm{KH}_{2} \mathrm{PO}_{4}$ & 1 & 1 & • & • & 1 & 1 & 1 & 1 & 1 & 1 & 1 & 1 & 1 \\
\hline${ }^{2} \mathrm{KCl}$ & 4 & 0.96 & 4.80 & • & 4 & 4 & 4 & 4 & 4 & 4 & 4 & 4 & 4 \\
\hline${ }^{3} \mathrm{CaCl}_{2} \cdot 2 \mathrm{H}_{2} \mathrm{O}$ & 1 & 1 & 1 & 1 & • & 1 & 1 & 1 & 1 & 1 & 1 & 1 & 1 \\
\hline${ }^{4} \mathrm{NH}_{4} \mathrm{H}_{2} \mathrm{PO}_{4}$ & - & • & $\bullet$ & 1 & • & • & $\bullet$ & $\bullet$ & • & $\bullet$ & - & • & • \\
\hline${ }^{5}\left(\mathrm{NH}_{4}\right)_{2} \mathrm{SO}_{4}$ & 2 & - & 2 & 2 & 2 & 2 & $\bullet$ & 2.09 & 2 & 2.01 & 2 & 2 & 2 \\
\hline${ }^{6} \mathrm{NaNO}_{3}$ & 1 & • & 1 & 0.91 & 1 & 1 & 1.34 & 0.98 & 1 & 1 & 1 & 1 & 1 \\
\hline${ }^{7} \mathrm{~K}_{2} \mathrm{SO}_{4}$ & - & 1 & • & • & • & • & $\bullet$ & • & • & • & - & • & • \\
\hline${ }^{8} \mathrm{MgCl} 2.6 \mathrm{H} 2 \mathrm{O}$ & 1 & 1 & 1 & 1 & 1 & • & 1 & 1 & 1 & 1 & 1 & 1 & 1 \\
\hline${ }^{9} \mathrm{FeCl}_{3} \cdot 6 \mathrm{H}_{2} \mathrm{O}$ & • & • & • & • & • & • & 1 & • & - & - & $\bullet$ & - & - \\
\hline${ }^{10} \mathrm{FeSO}_{4} \cdot 7 \mathrm{H}_{2} \mathrm{O}$ & 1 & 1 & 1 & 1 & 1 & 1 & - & • & 1 & 1 & 1 & 1 & 1 \\
\hline${ }^{11} \mathrm{H}_{3} \mathrm{BO}_{3}$ & 1 & 1 & 1 & 1 & 1 & 1 & 1 & 1 & • & 1 & 1 & 1 & 1 \\
\hline${ }^{12} \mathrm{MnCl}_{2} \cdot 4 \mathrm{H}_{2} \mathrm{O}$ & $\bullet$ & $\bullet$ & • & $\bullet$ & $\bullet$ & $\bullet$ & 1 & $\bullet$ & $\bullet$ & • & - & $\bullet$ & - \\
\hline${ }^{13} \mathrm{MnSO}_{4} \cdot \mathrm{H}_{2} \mathrm{O}$ & 1 & 1 & 1 & 1 & 1 & 1 & $\bullet$ & 1 & 1 & - & 1 & 1 & 1 \\
\hline${ }^{14} \mathrm{ZnSO}_{4} \cdot 7 \mathrm{H}_{2} \mathrm{O}$ & • & 1 & • & • & • & • & - & - & • & • & $\bullet$ & • & - \\
\hline${ }^{15} \mathrm{ZnCl}_{2}$ & 1 & • & 1 & 1 & 1 & 1 & 1 & 1 & 1 & 1 & $\bullet$ & 1 & 1 \\
\hline${ }^{16} \mathrm{CuCl}_{2}$ & $\bullet$ & • & $\bullet$ & $\bullet$ & - & • & 1 & $\bullet$ & $\bullet$ & • & $\bullet$ & $\bullet$ & $\bullet$ \\
\hline${ }^{17} \mathrm{CuSO}_{4} \cdot 5 \mathrm{H}_{2} \mathrm{O}$ & 1 & 1 & 1 & 1 & 1 & 1 & - & 1 & 1 & 1 & 1 & • & 1 \\
\hline${ }^{18} \mathrm{H}_{2} \mathrm{MoO}_{4} \cdot \mathrm{H}_{2} \mathrm{O}$ & 1 & 1 & 1 & 1 & 1 & 1 & 1 & 1 & 1 & 1 & 1 & 1 & $\bullet$ \\
\hline
\end{tabular}

Stock solutions $\left(\mathrm{g} . \mathrm{l}^{-1}\right):{ }^{1} 136.083 ;{ }^{293.198 ;}{ }^{37} 735.079 ;{ }^{4} 115.024 ;{ }^{5} 125.59 ;{ }^{6} 951.766 ;{ }^{73} 31.124 ;{ }^{8} 406.608 ;{ }^{9} 24.25 ;{ }^{10} 24.939 ;{ }^{11} 2.86004$; ${ }^{12} 1.80998 ;{ }^{13} 1.546 ;{ }^{14} 0.21099 ;{ }^{15} 0.09999 ;{ }^{16} 0.04001 ;{ }^{17} 0.0743 ;{ }^{18} 0.01999$. Note: (Cp.) complete treatment. (-) treatment without the relevant nutrient. $(\bullet)$ reagent not used in the relevant treatment.

Table 4 - Economic analysis of the cost to formulate one liter of culture solution according to Prado (2010), model A and model B.

Tabela 4 - Análise econômica do custo para formular um litro de solução de cultivo referente à Prado (2010), modelo A e modelo B.

\begin{tabular}{|c|c|c|c|c|c|}
\hline \multirow{2}{*}{ Treatment } & \multirow{2}{*}{ Prado (2010) } & \multirow{2}{*}{ Model A } & \multirow{2}{*}{ Model B } & \multicolumn{2}{|c|}{ Reduction according to Prado (2010) } \\
\hline & & & & Model A & Model B \\
\hline \multicolumn{6}{|c|}{ 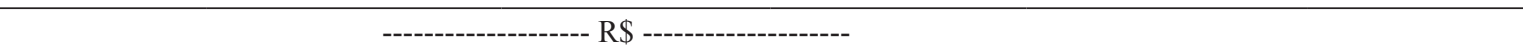 } \\
\hline Complete & 0.1052 & 0.0943 & 0.0780 & $10.37 \%$ & $25.84 \%$ \\
\hline$-\mathrm{N}$ & 0.0470 & 0.0470 & 0.0400 & $0.00 \%$ & $14.95 \%$ \\
\hline$-\mathrm{P}$ & 0.1069 & 0.0960 & 0.0797 & $10.21 \%$ & $25.42 \%$ \\
\hline$-\mathrm{K}$ & 0.0826 & 0.0722 & 0.0563 & $12.55 \%$ & $31.83 \%$ \\
\hline$-\mathrm{Ca}$ & 0.0835 & 0.0829 & 0.0666 & $0.66 \%$ & $20.16 \%$ \\
\hline$-\mathrm{Mg}$ & 0.0846 & 0.0760 & 0.0707 & $10.19 \%$ & $16.40 \%$ \\
\hline$-\mathrm{S}$ & 0.1400 & 0.1314 & 0.0880 & $6.16 \%$ & $37.19 \%$ \\
\hline$-\mathrm{Fe}$ & 0.1032 & 0.0923 & 0.0770 & $10.57 \%$ & $25.38 \%$ \\
\hline$-\mathrm{B}$ & 0.1051 & 0.0942 & 0.0780 & $10.38 \%$ & $25.85 \%$ \\
\hline$-\mathrm{Mn}$ & 0.1046 & 0.0937 & 0.0779 & $10.43 \%$ & $25.55 \%$ \\
\hline$-\mathrm{Zn}$ & 0.1052 & 0.0943 & 0.0780 & $10.37 \%$ & $25.84 \%$ \\
\hline$-\mathrm{Cu}$ & 0.1052 & 0.0943 & 0.0780 & $10.37 \%$ & $25.83 \%$ \\
\hline -Mo & 0.1052 & 0.0943 & 0.0780 & $10.37 \%$ & $25.84 \%$ \\
\hline Total & 1.2784 & 1.1629 & 0.9463 & $9.03 \%$ & $25.98 \%$ \\
\hline
\end{tabular}

Cerne, Lavras, v. 19, n. 2, p. 255-261, abr./jun. 2013 
Model B promoted a $25.98 \%$ cost reduction against what is suggested by Prado (2010) and a 18.63\% reduction in relation to model $\mathrm{A}$, and this logic is explained by the fact that model B works with 11 more choices of reagents than model $\mathrm{A}$. The treatment where $\mathrm{S}$ was omitted promoted the greatest cost reduction $(37.19 \%)$ due to replacement of magnesium nitrate $\left(x_{10}\right)$ with magnesium chloride $\left(x_{21}\right)$ and to use of sodium nitrate $\left(x_{18}\right)$ as source of $\mathrm{N}$. Around 8 treatments attained savings of $25.90 \%$, and the smallest reduction was $14.95 \%$. However, the $14.95 \%$ reduction in model B was greater than the maximum attained in model A (12.55\%).

A similar behavior was noted in all three cases regarding the costs of treatments omitting $\mathrm{N}$ and $\mathrm{S}$, the first generating lower costs and the second generating higher costs among treatments. N-supplying reagents entail high cost for formulation of nutrient solutions, which explains the low cost of the treatment omitting N, when such reagents are not present. The high cost of the treatment omitting $\mathrm{S}$ is explained by the large number of reagents with this nutrient in their composition, 8 in total, thus forcing use of a limited number of reagents which, in turn, constitute a high-costing group.

Table 5 provides costs of reagent acquisition for conducting the simulated experiment, showing that values could be as high as around $\mathrm{R} \$ 2,000.00$, a significant sum considering the intended purpose of the research.

Table 5 - Comparative analysis of total cost of reagents $(\mathrm{R} \$)$ in conducting the simulated experiment via the missing element technique.

Tabela 5-Análise comparativa para o custo total dos reagentes (R\$) na condução do experimento simulado via técnica do elemento faltante.

\begin{tabular}{|c|c|c|c|}
\hline \multirow{2}{*}{ References } & \multicolumn{3}{|c|}{ Total cost of reagents $(\mathrm{R} \$)$} \\
\hline & Plot & Replicate & Experiment \\
\hline \multicolumn{4}{|c|}{ 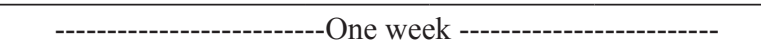 } \\
\hline Prado (2010) & 3.84 & 15.34 & 122.72 \\
\hline Model A & 3.49 & 13.95 & 111.64 \\
\hline Model B & 2.84 & 11.36 & 90.84 \\
\hline Savings in Model A & 0.65 & 2.60 & 20.80 \\
\hline Savings in Model B & 1.00 & 3.99 & 31.88 \\
\hline \multicolumn{4}{|c|}{--------------------------Sixteen weeks ------------------------ } \\
\hline Prado (2010) & 61.36 & 245.44 & 1963.55 \\
\hline Model A & 55.82 & 223.28 & 1786.21 \\
\hline Model B & 45.42 & 181.68 & 1453.46 \\
\hline Savings in Model A & 10.40 & 41.59 & 332.75 \\
\hline Savings in Model B & 15.94 & 63.76 & 510.08 \\
\hline
\end{tabular}

The cost reduction promoted by the LP mathematical models proposed in this work reached over $\mathrm{R} \$ 500.00$ (model B) per experiment. These savings could be the determining factor for the viability or not of a research project, and help shift the focus of research onto other priorities such as sampling intensity, increasing the number of vases per replicate (plot) or the number of replicates, or else extend the experiment duration (weeks) (Table 6).

Table 6 - Savings generated by models A and B based on the simulated experiment, observing number of plots, replicates and weeks.

Tabela 6 - Economia gerada pelos modelos A e B baseado no experimento simulado observando o número de parcelas, repetições e semanas.

\begin{tabular}{lcc}
\hline Category & Model A & Model B \\
\hline Plots & 0.75 & 1.40 \\
Replicates & 1.49 & 2.81 \\
Weeks & 2.98 & 5.62 \\
\hline
\end{tabular}

The savings of $\mathrm{R} \$ 332.75$ in model A could allow addition of an extra replicate in the experimental design or else prolong the duration of the experiment for another three weeks. As for model B, the generated savings of R\$ 510.08 could allow addition of an extra vase per replicate (plot), or two replicates, or else 5 weeks conducting the experiment. Here, savings generated by models A or B and applied to the experiment could bring benefits, for instance, improve experimental accuracy with increased sample intensity.

Considering the savings generated by model B only, and based on 30 experiments per year, a reduction of $\mathrm{R} \$ 15,302.40 /$ year in expenditure would be achieved, something real and practical in most laboratories of research institutions and universities. This statement is intended to serve as a reference for planning of laboratories exploring mineral nutrition of plants, where the number of experiments being conducted in a short time span is expressive.

That said, although linear programming has been known for a while, it has not been widely used for assisting with decision-making in laboratory planning, yet it could potentially make a difference where costs are concerned, bringing benefits to research.

\section{CONCLUSIONS}

The models formulated via LP proved to be effective in minimizing the acquisition cost of reagents

Cerne, Lavras, v. 19, n. 2, p. 255-261, abr./jun. 2013 
for generating nutrient solutions in experimentation with plant nutrients, observing the desired concentrations of each nutrient. However, real trials should be conducted to check the recommendation suggested by the mathematical models for viability. The cost reduction was $9.03 \%$ in model A and $25.98 \%$ in model $\mathrm{B}$, based on the reference table provided by Prado (2010).

The recommendation tables for formulating experiments on nutrient deficiency with the missing element technique can be easily generated based on results obtained in models A and B.

\section{REFERENCES}

BERGER, R.; CARNIERI, C.; LACOWICZ, P. G.; BRASIL, A. A. Minimização de custos de transporte florestal com a utilização da programação linear. Floresta, Curitiba, v. 33, n. 1, p. 53-62, 2003.

GOLDBARG, M.; LUNA, H. Otimização combinatória e programação linear: modelos e algoritmos. 2. ed. Rio de Janeiro: Elsevier, 2005. 518 p.

LIEBIG, J. von. Chemistry and its application to agriculture and physiology. London: Tauylor and Walton, 1840.

MAFFEIS, A. R.; SILVEIRA, R. L. V. de A.; BRITO, J. O. Reflexos das deficiências de macronutrientes e boro no crescimento de plantas, produção e qualidade de óleo essencial em Eucalyptus citriodora. Scientia Forestalis, Piracicaba, n. 57, p. 87-98, jun. 2000.

MARQUES, T. C. L. L. de S. e M.; CARVALHO, J. G. de; LACERDA, M. P. C.; MOTA, P. E. F. da. Crescimento inicial do Paricá (Schizolobium amazonicum) sob omissão de nutrientes e de sódio em solução nutritiva. Cerne, Lavras, v. 10, n. 2, p. 184-195, jul./dez. 2004.
NOVAIS, R. F.; ALVAREZ, V. H. V.; BARROS, N. F.; FONTES, R. L. F.; CANTARUTTI, R. B.; NEVES, J. C. L. Fertilidade do solo. Viçosa, MG: SBCS, 2007. 1017 p.

OLIVEIRA, A. J.; GARRIDO, W. E.; ARAÚJO, J. D.; LOURENÇO, S. (Coord.). Métodos de pesquisa em fertilidade do solo. Brasília: EMBRAPA-SEA, 1991. 392 p.

PRADO, R. de M. Diagnose de deficiência nutricional em culturas. Jaboticabal: UNESP, 2010. Disponível em: $<$ http:// www.nutricaodeplantas.agr.br/site/downloads/unesp_jaboticabal/ trabalho_pratico.pdf > . Acesso em: 25 abr. 2011.

RODRIGUEZ, L. C. E.; LIMA, A. B. H. P. M. de. A utilização da programação linear na determinação de uma estratégia ótima de reforma de um talhão florestal. IPEF, Piracicaba, n. 31, p. 47-53, dez. 1985.

SILVA, E. de B.; GONÇALVES, N. P.; PINHO, P. J. de. Limitações nutricionais para crescimento de mudas de umbuzeiro em Latossolo Vermelho distrófico no Norte de Minas. Acta Scientiarum, Maringá, v. 27, n. 1, p. 55-59, jan./ mar. 2005.

SILVA, G. F. da; LEITE, H. G.; SILVA, M. L. da; RODRIGUES, F. L.; SANTOS, H. do N. Problemas com o uso de programação linear com posterior arredondamento da solução ótima, em regulação florestal. Revista Árvore, Viçosa, v. 27, n. 5, p. 677688, set./out. 2003.

TAIZ, L.; ZEIGER, E. Fisiologia vegetal. 3. ed. Porto Alegre: Artmed, 2004. 719 p.

WALLAU, R. L. R. de; BORGES, A. R.; ALMEIDA, D. R. de; CAMARGOS, S. L. Sintomas de deficiências nutricionais em mudas de mogno cultivadas em solução nutritiva. Cerne, Lavras, v. 14, n. 4, p. 304-310, out./dez. 2008.

Cerne, Lavras, v. 19, n. 2, p. 255-261, abr./jun. 2013 
